\title{
Third Order Optical Nonlinearities and Spectral Characteristics of Methylene Blue
}

\author{
Velloli Sindhu Sukumaran ${ }^{1^{*}}$, Alkondan Ramalingam ${ }^{2}$ \\ ${ }^{1}$ Central Institute of Plastics and Engineering Technology Guindy, Chennai, India \\ ${ }^{2}$ Centre for Laser Technology, Department of Physics, Anna University, Chennai, India \\ E-mail: sindhusukumaran@yahoo.co.in \\ Received May 24, 2011; revised July 6, 2011; accepted July 26, 2011
}

\begin{abstract}
We have investigated third order nonlinear optical properties and spectral characteristics of methylene blue dye in both polymer and liquid mixtures. The spectral characteristics of the dye is studied by recording the absorption and fluorescence spectra of the dye doped in poly(methylmethacrylate) modified with additive n-butyl acetate(nBA) and the dye in MMA and nBA (liquid mixture). The spectral results of the dye doped polymer rod are compared with dye in liquid Mixture. The nonlinear measurements of the dye in liquid and polymer medium were performed using $\mathrm{CW}$ He-Ne laser of wavelength $632.8 \mathrm{~nm}$ by employing z-scan technique. The dye methylene blue showed a negative nonlinear refractive index.
\end{abstract}

Keywords: Methylene Blue, Polymer, Solid Dye Laser, nBA, MMA, Spectral Characteristics, Nonlinear Refractive Index, Polymer Thin Film

\section{Introduction}

The protection of sensitive optical equipment has been the focus of much attention in recent times. Of great importance is the protection of human eye from potentially harmful intense beams. A large number of compounds have been synthesized to realize nonlinear susceptibilities far larger than the inorganic optical material [1]. In this paper we report the synthesis, characterization and nonlinear optical properties of methylene blue dye doped polymer and compare it with dye doped monomer. Nonlinear optical properties of polymer solution was studied by means of a z-scan set-up. Nonlinear optical effects can be employed for the design and performance of optical limiter. It has been showed that optical limiting can be used for the protection of eyes and sensors from intense lasers [2].

Solid matrix used as lasers get rid of many of the common problems associated with static or flowing liquid systems. The most frequently used polymeric material is polymethylmethacrylate (PMMA) [3]. Review of literature showed most of the work on dye-doped polymers was done with rhodamine dyes [4] and pyromethane dyes [5]. Some work was reported on coumarin dyes [3] and on dye IR140 [6].

In this paper, the fabrication of dye methylene blue doped polymer rods and films, its spectral parameters and the study of nonlinear refractive index under the HeNe laser excitation are reported. The properties of the dyes in liquid medium are compared with that in the solid matrix.

\section{Experimental Procedure}

\subsection{Synthesis of Dye-Doped Polymer Rods and thin Films}

Methylene blue, a phenothiazine dye, supplied by Exciton, USA, is chosen for the study. The molecular structure of the dye is shown in Figure 1. Thin layer choromatography (TLC) test confirms the absence of any impurities in the dye. Methyl methacrylate (MMA) is used as a monomer for synthesizing dye doped polymer film. Initial MMA compositions are cleared of foreign inclusions. Spectroscopic grade n-butyl acetate (nBA) is used

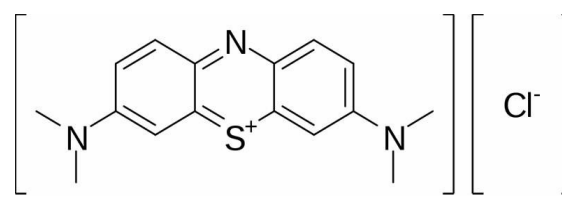

Figure 1. Molecular Structure of Methylene blue. 
as additive. The dye doped polymer film (DDP) and rod are synthesized using thermal bulk free radical polymerization [7] of dye concentration $0.05 \times 10^{-3} \mathrm{M}$. The internal optical qualities of polymer rods and film are checked by passing the laser beam of $5 \mathrm{~mW}$ He-Ne laser $(632.8 \mathrm{~nm})$ through these rods. No dispersion or distortion of the He-Ne laser beam was observed.

\subsection{Spectral Characteristics, Life Time, Quantum Yield}

The UV-VIS absorption and fluorescence spectrum of the dye in liquid medium and the solid matrix (PMMA + nBA) was obtained using Hitachi U2000 spectrophotometer and Hitachi F2000 spectrofluorometer respectively. These spectra are shown in Figures 2 and 3. The fluorescence lifetime is studied by using the picosecond time correlated single photon counting technique and employing analytical deconvocation methods. Quantum yields [8] are calculated using Rhodamine $6 \mathrm{G}$ in ethanol as the fluorescence standard with refractive index and differential absorption correction. The spectral parameters such as absorption peak wavelength, molar extinc-

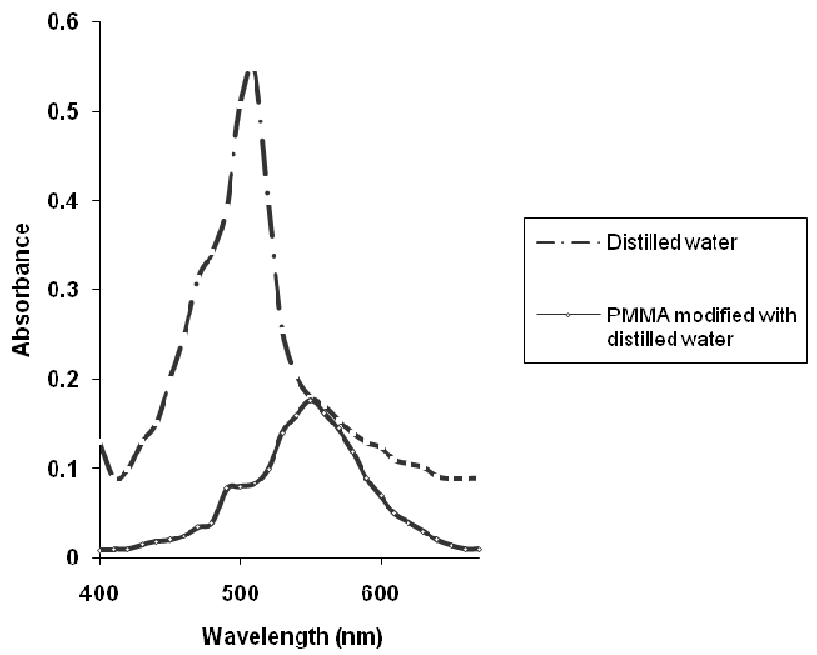

Figure 2. Absorption spectra of dye Methylene blue in PMMA + nBA and MMA + nBA. tion coefficient $(€)$, band width $\left(\Delta \ddot{v}_{1 / 2}\right)$, oscillator strength (F), fluorescence peak wavelength, full width at half maximum (FWHM), Stoke's shift of the dyes and the calculated lifetime $\left(\tau\right.$ f) quantum yield $\left(\emptyset_{\mathrm{f}}\right)$, radiative $\left(\mathrm{K}_{\mathrm{r}}\right)$ and non-radiative $(\mathrm{Knr})$ decay constants of the dyes are shown in Table 1.

\subsection{Nonlinear Studies}

The closed z-scan [9] set up, developed by Sheik Bahae et al. is used to characterize the nonlinear optical properties of the dye in different mixtures. It is based on intensity dependent refractive index and includes variation of refractive index as a function of incident beam irradiance of the sample. The set up is shown in Figure 4. A Gaussian beam from He-Ne laser ( $\lambda$ : $632.8 \mathrm{~nm}$, power: 10 $\mathrm{mW}$ ) was focused by a convex lens of focal length $20 \mathrm{~cm}$ and passed through the sample. The sample is scanned through the beam, the far field profile shows intensity variation across the beam profile, which is recorded through an aperture using photo detector fed to the digital power meter (Field Master GS- coherent). The figure provides not only the magnitude of real and imaginary

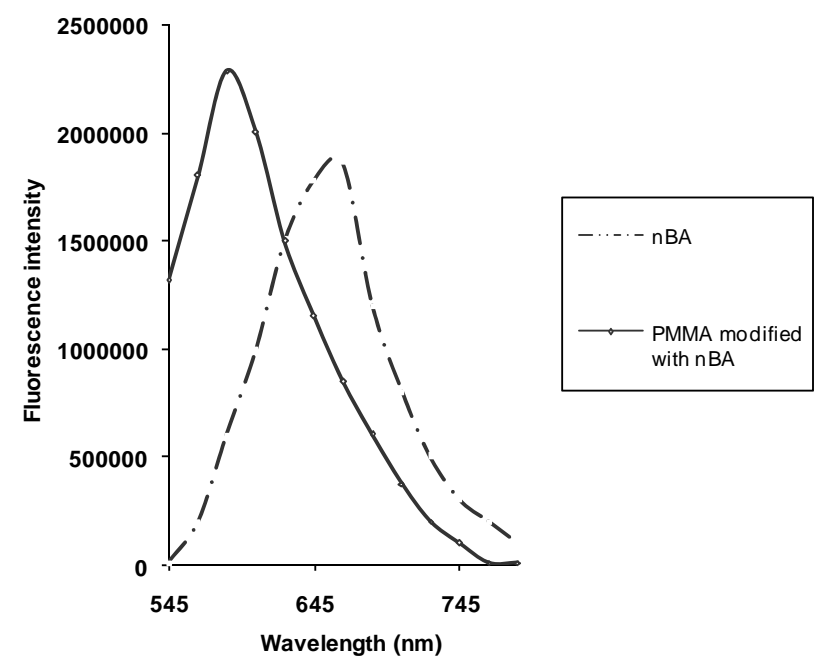

Figure 3. Fluorescence spectra of dye Methylene blue in PMMA + nBA and MMA + nBA.

Table 1. Spectral characteristics, quantum yield, lifetime, radiative and non-radiative decay constants of the dye Methylene blue.

\begin{tabular}{|c|c|c|c|c|c|c|c|}
\hline \multirow{2}{*}{ Solvent/Medium } & \multicolumn{3}{|c|}{ Absorption spectra } & \multirow[b]{2}{*}{$\begin{array}{l}\text { Oscillator Strength } \\
\text { f } 10^{-24} \mathrm{~L} \mathrm{~mol}^{-1} \mathrm{~cm}^{-2}\end{array}$} & \multicolumn{3}{|c|}{ Fluorescence spectra } \\
\hline & $\begin{array}{l}\text { Peak wavelength } \\
\text { in } \mathrm{nm}\end{array}$ & $\begin{array}{c}\varepsilon 10^{4} \mathrm{~L} \\
\mathrm{~mol}^{-1} \mathbf{c m}^{-1}\end{array}$ & $\begin{array}{l}(\Delta v)_{1 / 2} \\
\mathbf{c m}^{-1}\end{array}$ & & $\begin{array}{l}\text { Peak wavelength } \\
\text { in } \mathrm{nm}\end{array}$ & $\begin{array}{l}\text { FWH } \\
\text { M nm }\end{array}$ & $\begin{array}{c}\text { Stoke's } \\
\text { shift } \text { cm }^{-1}\end{array}$ \\
\hline Distilled water & 600 & 0.4 & 2797.2 & 0.4844 & 662 & 98 & 1560.9 \\
\hline $\begin{array}{l}\text { PMMA modified with } \\
\text { Distilled water }\end{array}$ & 555 & 0.352 & 2541.3 & 0.3873 & 588 & 107.5 & 1011.2 \\
\hline
\end{tabular}


parts of the nonlinear susceptibility, but also the sign of

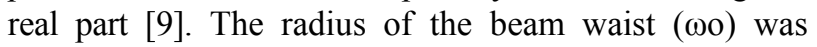
$75 \mu \mathrm{m}$ with a Rayleigh range of $27.91 \mathrm{~mm}$. Care is taken that the absorber is not saturated. The film is more valuable in application than the solution and the discrepancy between films and solutions will greatly affect the behavior of molecules, which will have an obvious influence on their nonlinear properties.

\section{Results}

The analysis z scan curve of methylene blue showed that dye has a negative (self-defocusing) nonlinearity. The peak followed by a valley - normalized transmittance curve obtained from the closed $\mathrm{z}$ scan data, indicates the sign of refraction nonlinearity is negative, i.e., Self defocusing effect. The self defocusing effect is due to local variation of refractive index with temperature. The defocusing effect of the dye in liquid medium and in polymer film is shown in Figures 5 and 6, is attributed to a thermal nonlinearity resulting from absorption of $632.8 \mathrm{~nm}$.

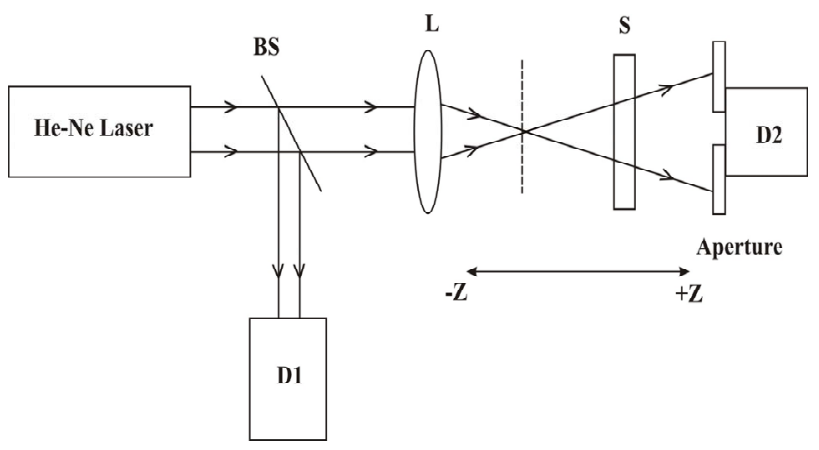

Figure 4. Z scan set-up.

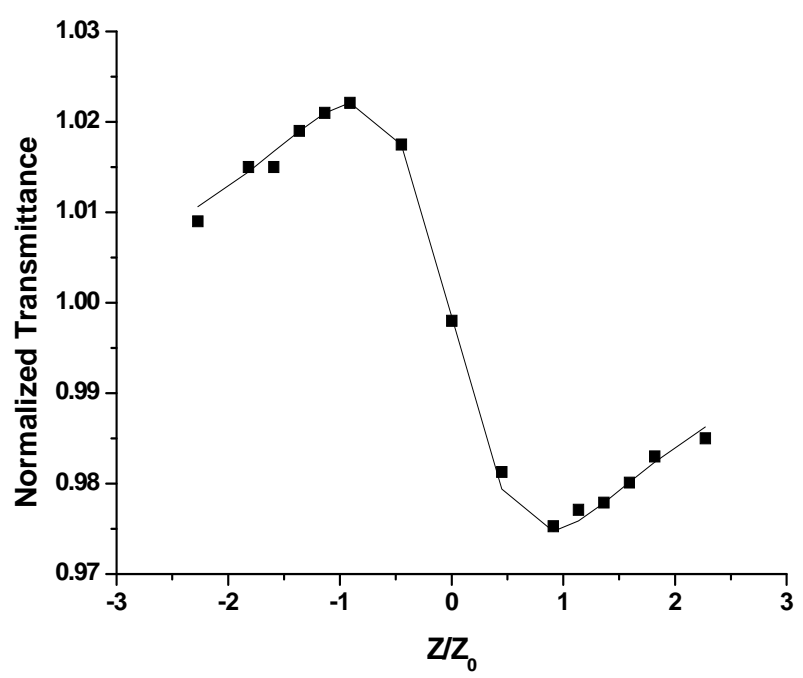

Figure 5. Measured z-scan of the dye Methylene blue. The solid line is the calculated result with $\Delta \emptyset_{0}=-0.95$.

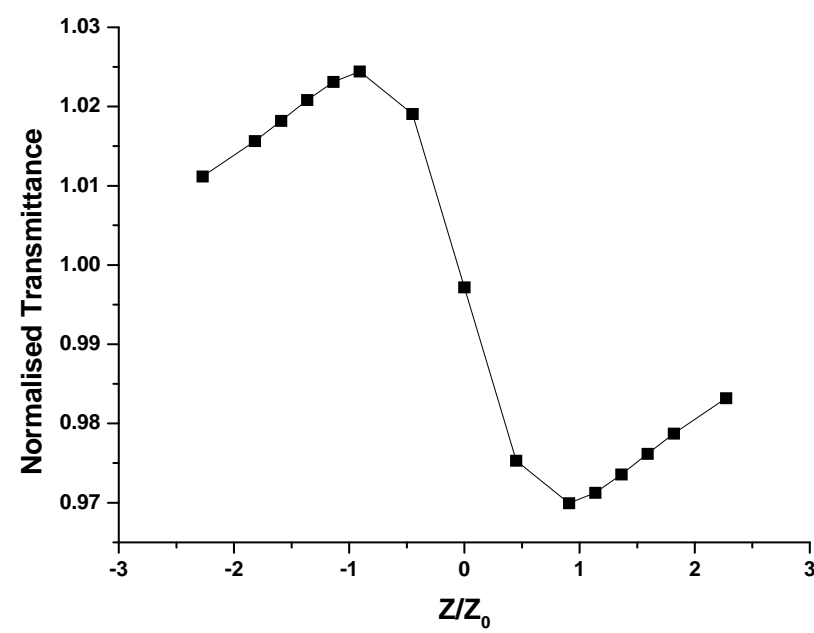

Figure 6. Measured $Z$ scan of the dye methylene blue doped polymer film. The solid line is the calculated result with $\Delta \varphi_{0}$ $=-\mathbf{0 . 1 3}$.

The localized absorption of tightly focused beam propagating through the absorbing dye medium produces a spatial distribution of temperature in the dye solution and, consequently, a spatial variation of refractive index that act as a thermal lens resulting in phase distortion of the propagating beam. The z-scan signature for the dye in liquid medium gives the value of the transmission from peak to valley $\left(\mathrm{T}_{\mathrm{p}-\mathrm{v}}\right)$ as 0.0468 , which shows an index change of $\left\langle\Delta \mathrm{n}_{\mathrm{o}}\right\rangle=-1.907 \times 10^{-6}$. This value of $\left\langle\Delta \mathrm{n}_{\mathrm{o}}\right\rangle$ corresponds to the value of $\gamma=-1.05159 \times 10^{-12} \mathrm{~m}^{2} / \mathrm{W}$. The z-scan signature for the dye Methylene blue in solid matrix (polymer film) gives the value of the transmission from peak to valley $\left(\mathrm{T}_{\mathrm{p}-\mathrm{v}}\right)$ as 0.0537 , which gives an index change of $-1.362 \times 10^{-6}$. This gives $\gamma=-1.20295 \times$ $10^{-12} \mathrm{~m}^{2} / \mathrm{W}$

\section{Acknowledgements}

Authors wish to acknowledge National Centre for Ultra fast Processes, University of Madras for extending the facilities available in their centre.

\section{References}

[1] T. Malli and T. Kar, "Growth and Characterization of Nonlinear Optical L-Arginine Dyhydrate Single Crystals," Journal of Crystal Growth, Vol. 285, 2005, pp. 178-182. doi:10.1016/j.jcrysgro.2005.08.025

[2] Q. S. Li, C. L. Liu, L.Y. Zang, Q. H. Gong, X. L. Yu and C. B. Cao, "Broadband Optical Limiting in the Suspensions of Lead Sulfide Nanoparticles," Laser Physics, Vol. 18 , No. 4, 2008, pp. 434-437. doi:10.1134/s11490-008-4013-3

[3] G. Somasundaram and A. Ramalingam, "Gain Studies of 
Coumarine 1 Dye Doped Polymer Laser," Journal of Lumin, Vol. 90, 2000, pp. 1-5.

doi:10.1016/S0022-2313(99)00608-0

[4] A. Costela, I. Garcia-Marino, J. M. Figuera, F. Amat Guerri and R. Sastre, "Recent Developments in Polymeric Solid-State Dye Laser," Recent Developments in Physical Chemistry, Vol. 1, 1997, pp. 125-131.

[5] M. Canva, P. Georges, J. F. Perelgritz, A. Brum and F. Chaput, "Perylene- and Pyrromethene-Doped Xerogel for a Pulsed Laser," Applied Optics, Vol. 34, No. 3, 1995, pp. 428-431. doi:10.1364/AO.34.000428

[6] U. Tripathy, R. Justin Rajesh, P. B. Bisht and A. Subrahamanyam, "Optical Nonlinearity of Organic Dyes as Studied by z- Scan and Transient Grating Techniques," Chemical Science, Vol. 114, No. 6, 2002, pp. 557-564.

\section{doi:10.1007/BF02708850}

[7] A. Costela, I. Garcia-Marino, J. M. Figuera, F. AmatGuerri, J. Barrosoand and R. Shastre, "Solid-State Dye Laser Based on Coumarine 540 A Doped Polymeric Matrices," Optics Communications, Vol. 130, No. 1, 1996, pp. 44-50. doi:10.1016/0030-4018(96)00220-9

[8] J. N. Demas and G. A. Crosby, "The Measurement of Photoluminescence Quantum Yields: A Review," Journal of Physical Chemistry, Vol. 75, 1971, pp. 991-1028. doi:10.1021/j100678a001

[9] R. K. Rekha and A. Ramalingam, "Optical Nonlinear Properties and Optical Limiting Effect of Metanil Yello," American Journal of Engineering and Applied Sciences, Vol. 2, No. 2, 2009, pp. 285-291.

doi:10.3844/ajeassp.2009.285.291 